\title{
Hereditary Diffuse Gastric Cancer: A Family Diagnosis and Treatment
}

\author{
Adedayo A. Onitilo, MD, MSCR, FACP; Govinda Aryal, MD; and Jessica M. Engel, MS, RN
}

\begin{abstract}
Hereditary diffuse gastric cancer (HDGC) is a rare cancer representing approximately $2 \%$ of all gastric cancers. It is caused by $\mathrm{CDHI}$ gene mutations, inherited in an autosomal dominant fashion, that affect the function of E-cadherin. Approximately 38\% of HDGC families have a $\mathrm{CDHI}$ gene mutation. With an estimated $75 \%$ penetrance rate, carriers are at high risk for HDGC. We describe the case of a Caucasian male of German-Russian ancestry, carrying a $\mathrm{CDHI}$ gene mutation, who survived for 18 months after being diagnosed with HDGC. The results of genetic testing undergone by his family members are also reported, along with a review of the current literature. Since surveillance methods for HDGC are ineffective and unreliable, total prophylactic gastrectomy is advised for individuals with the gene mutation. Additionally, a diagnosis of HDGC should lead to genetic evaluation of family members followed by preventative measures.
\end{abstract}

Keywords: CDHI gene mutation; E-cadherin; Genetic testing; High disease expression; Prophylactic gastrectomy

Corresponding Author:

Adedayo A. Onitilo, MD, MSCR, FACP

Marshfield Clinic Weston Center

350 I Cranberry Boulevard

Weston, WI 54476

Tel: (7I5) 393-I400

Fax: (715) 393-1399

Email: onitilo.adedayo@marshfieldclinic.org

Received: December 14, 2011

Ist Revision: March 28, 2012

2nd Revision: April 12, 2012

Accepted: April 18, 2012

doi: $10.3121 / \mathrm{cmr} .2012 .107 \mid$ astric cancer is a common cause of cancer-related death worldwide, leading to approximately 700,000 deaths per year. ${ }^{1,2}$ However, it is one of the less common types of cancer diagnosed in the United States, with an estimated 21,520 new diagnoses and 10,340 deaths in 2011 as reported by the National Cancer Institute. $^{3}$ One type of gastric cancer known as hereditary diffuse gastric cancer (HDGC) is relatively uncommon, representing about $2 \%$ of all cases of gastric cancer. ${ }^{4-7}$

HDGC is a rare autosomal dominant genetic disorder typically caused by a CDH1 germline mutation, resulting in loss of function of the E-cadherin cell adhesion protein. ${ }^{2,7}$ Early detection of HDGC is difficult because the cancer is located submucosally. Consequently, prognosis is poor, mainly because there is lack of effective treatment for patients who become symptomatic. Despite the rarity of diagnosis, the impact on the family may be quite dramatic, especially as multiple family members may be affected, and at a young age, because CDH1 gene mutations have a penetrance of about $75 \%{ }^{4,7,8}$ However, a patient's diagnosis may be potentially lifesaving for other family members because genetic testing can be performed to detect the mutation, and then a prophylactic gastrectomy can be done, greatly reducing the risk of developing HDGC.

We herein describe the case of a Caucasian male of German-Russian ancestry who carries a CDH1 gene mutation and was diagnosed with HDGC. We report the results of his family members' genetic testing and present a review of the current literature. 

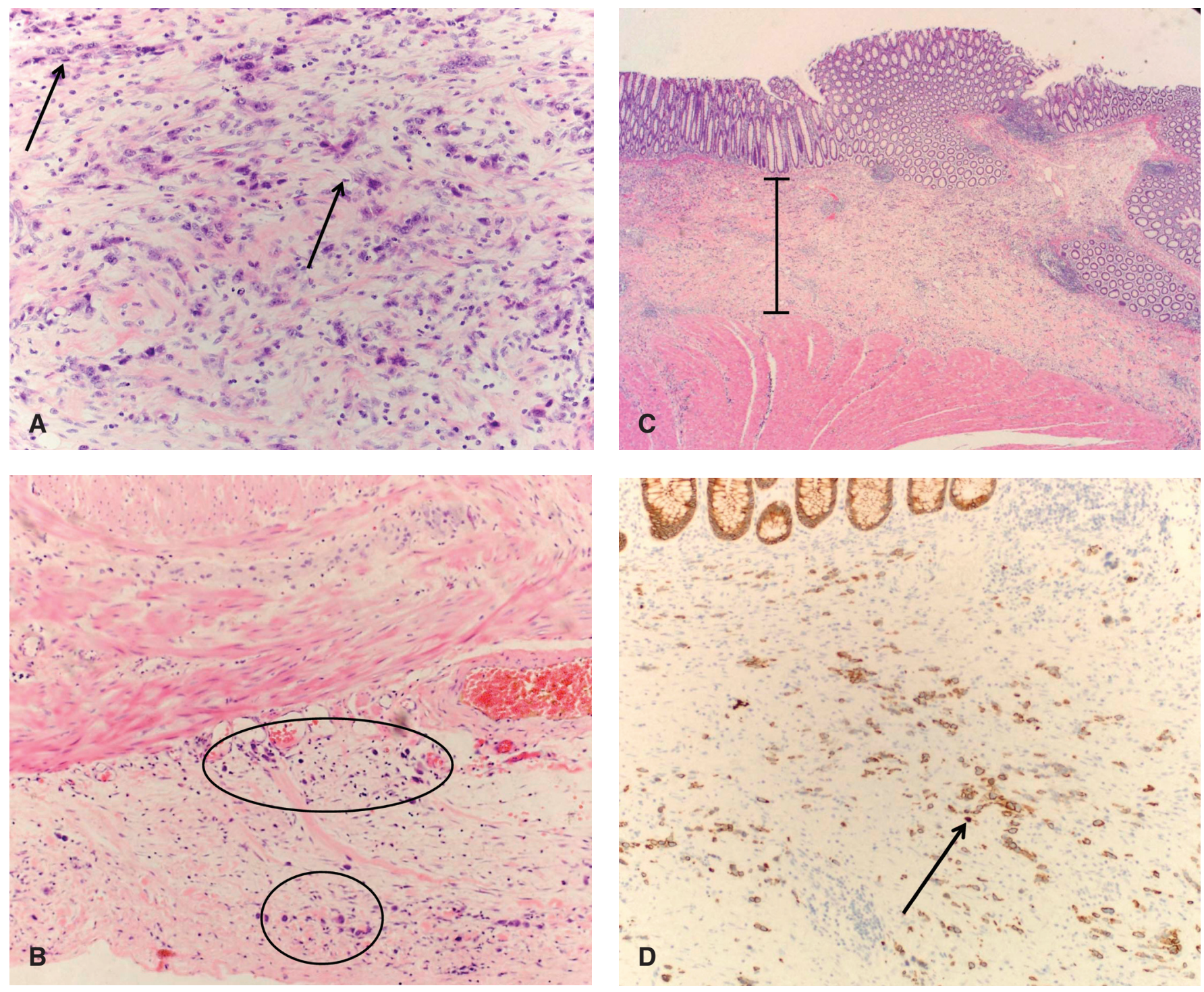

Figure 1. The metastatic adenocarcinoma, grade III/III; (A) arrows identify a couple of the many signet ring cells (H\&E, x200); (B) circles identify the involved colonic serosa (H\&E, x100); (C) the expanded submucosa as indicated by the bar (H\&E, x20); (D) cytokeratin 7 stain better highlights the metastatic adenocarcinoma. Arrow identifies a prominent cytokeratin 7 stained cell (x100).

\section{Case Presentation}

A man, age 56 years, from the mid-Western United States and of Russian-German ancestry, presented with epigastric and right upper quadrant abdominal pain accompanied by difficulty swallowing. A right upper quadrant ultrasound showed cholelithiasis. An esophagogastroduodenoscopy was performed about 3 weeks later and showed changes suggestive of reflux esophagitis. A gastric biopsy showed only chronic inflammation with no malignancy. His symptoms continued for about one month, and a laparoscopic cholecystectomy was performed with pathology again negative for malignancy.

The patient's symptoms worsened, and the evaluation continued. About three months after initial presentation, a computed tomography (CT) of the abdomen showed likely bowel obstruction at the level of the proximal descending colon, although colonoscopy was negative. During exploratory laparotomy, a significant stricture of the descending colon was found with thickening and induration of adjacent tissues (spleen, tail of pancreas, mesentery, and retroperitoneum). He underwent a left hemicolectomy. Due to significant edema at the splenic hilum, a splenectomy was also done. The gross specimen was $30 \mathrm{~cm}$ long with a circumference of $4.5 \mathrm{~cm}$ on one end and $11 \mathrm{~cm}$ on the opposite end, narrowing to $2.5 \mathrm{~cm}$ in an area $9 \mathrm{~cm}$ from the $4.5 \mathrm{~cm}$ circumference end, with an attached $15 \mathrm{~cm} \times 6.5 \mathrm{~cm} \times 0.5 \mathrm{~cm}$ portion of the omentum and spleen.

Tissue pathology from the colon showed stage IV metastatic adenocarcinoma, grade III/III, possibly gastric, diffuse type with scattered signet ring cells (figure 1A) extensively involving the mesentery, colonic serosa (figure 1B), muscularis propria, submucosa with associated prominent fibrosis and 
collagenization (figure 1C), and omentum with focal involvement. There was no evidence of mucosal involvement, margins were negative, two small mesenteric lymph nodes were negative, and the spleen and accessory spleen were negative. The cancer appeared aggressive, extending into, rather than arising from, the colon. Immunostaining indicated keratin cocktail positive, cytokeratin 7 positive, and cytokeratin 20 negative (figure 1D), supporting the pathological diagnosis. Additional staining showed epidermal growth factor receptor positive, CD117 negative, and human epidermal growth factor receptor 2 negative (value of zero).

The patient had postoperative complications which delayed his care. One month later, upper endoscopy was performed showing gastritis, white-based erosions in the gastric body, and a $4 \mathrm{~mm}$ to $5 \mathrm{~mm}$ shallow ulceration with whitish exudate in the middle of the corpus on the lesser curvature side. Biopsy of this area showed poorly differentiated signet ring adenocarcinoma which solidified the diagnosis of metastatic gastric cancer.

Post-surgical positron emission tomography (PET)/CT scan was negative for metastatic disease, but showed post-surgical changes and changes related to an abscess. The patient developed a subphrenic abscess secondary to complications of anastomotic failure accompanied by weight loss due to significant malnutrition. He underwent a bowel resection with ostomy. Interestingly, the pathology from this surgery did not show any malignancy; biopsy of the lymph nodes and omentum were negative. Repeat PET/CT after one month showed the abscess accompanied by gastric wall thickening.

Genetic testing for HDGC was suggested, as his mother had died of gastric cancer at age 51. DNA sequencing results showed heterozygosity for the CDH1 gene mutation (CDH1 $1003 \mathrm{C}>\mathrm{T}$; $\arg 335$ ter). Treatment options were entertained; however, it was determined that he was not a candidate for gastrectomy or other surgery. Once stable, he began chemotherapy with docetaxel, oxaliplatin, and 5-flurouracil. Two months after diagnosis, cycle 1 was given, nine cycles total. He developed peripheral neuropathy with this regimen, and for three additional cycles received continuous infusion 5-flurouracil (5-FU) alone.

Follow-up esophagogastroduodenoscopy showed stable appearance, but large, tortuous folds with exudates and small ulcerations and severe reflux esophagitis. Biopsy showed chronic inflammation but was negative for malignancy. Follow-up CT scan was stable with residual non-specific gastric wall thickening. The only new finding was a small amount of ascetic fluid. Eight months post-diagnosis, additional chemotherapy commenced with CPT-11/ bevacizumab/continuous infusion 5-FU. He received nine cycles over five months. Toward the end of this chemotherapy course, symptoms of uncontrolled weight loss returned. A CT scan showed progressive disease with a posterior $4.1 \mathrm{~cm}$ gastric body mass with interval necrosis and generalized gastric wall thickening, stable peritoneal thickening, and

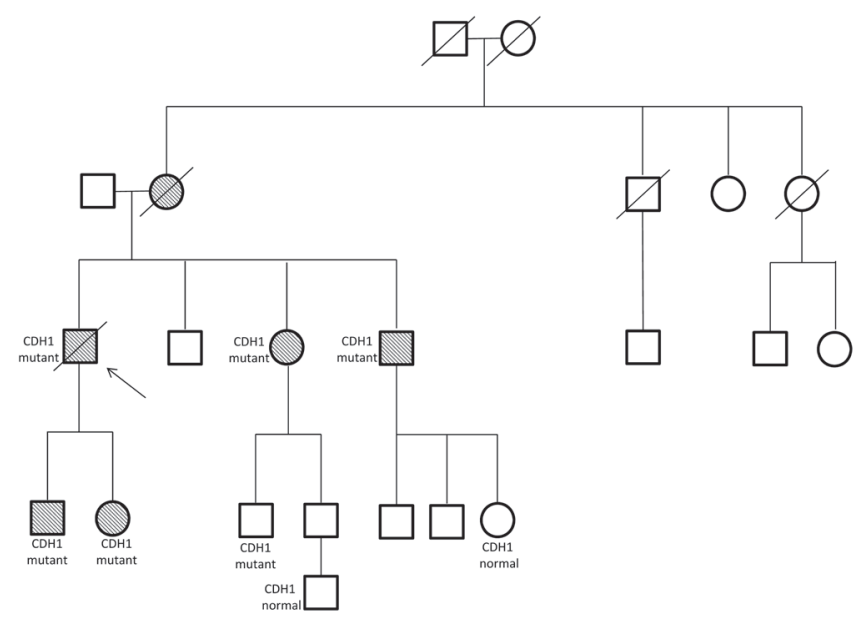

Figure 2. Family pedigree. Shaded circles and squares indicate gastric cancer. The arrow points to our patient (age 56 years).

enlarged right supradiaphragmatic lymph node. His chemotherapy regimen was changed to docetaxel and continuous infusion 5-FU. He received four cycles over two months. He developed pneumonia which delayed further treatment.

Nineteen months after the initial diagnosis of metastatic gastric cancer, he developed symptoms of nausea and vomiting. A CT scan showed possible small bowel obstruction and peritoneal fluid accumulation. Cytology was positive for adenocarcinoma. He was treated conservatively and showed symptom improvement. Chemotherapy was resumed with one cycle of epirubicin, oxaliplatin, and 5-FU given; however, about two weeks later the patient died.

\section{Genetic Testing of Family Members}

Although this patient was diagnosed with a terminal cancer at an early age and his prognosis was poor, his advocacy for genetic testing led to potentially life-saving measures for his family members (figure 2 ). He was the only family member diagnosed and treated at our facility; all others were treated elsewhere. Therefore, only limited information is available; pathology for his family members is not available. His sister (age 50) and a brother (age 48) tested positive for the CDH1 gene mutation. Soon after, his sister underwent prophylactic gastrectomy; two sites of gastric cancer were found, and a diagnosis of poorly differentiated signet ring adenocarcinoma was made. At age 49 , his brother was diagnosed with gastric cancer, but it is not known what decisions he made. Another brother (at the age of 48) was tested, but chose to not disclose the results.

The patient's son and daughter were also tested. His son was tested at age 25, and has the CDH1 gene mutation. At age 26, he was found to have multiple sites of cancer on a screening endoscopy and stomach biopsy. He underwent prophylactic gastrectomy, and the surgical pathology showed six T1a tumors. A T1a staged tumor is one that has invaded the lamina 
propria and muscularis mucosae. The daughter (age 24) also tested positive for the CDH1 gene mutation. She had three screening endoscopies over 18 months, with all biopsies negative for malignancy. She then underwent prophylactic gastrectomy at the age of 26 , and the pathology was positive for gastric cancer; at least 25 surgical pathology specimens showed 32 sites of T1a tumor.

\section{Discussion}

The CDH1 gene is located on chromosome 16 and encodes E-cadherin. ${ }^{9,10}$ E-cadherin is a calcium-dependent cell-cell adhesion protein that plays a role in the maintenance of cell differentiation and the normal architecture of epithelial cells, thereby functioning as a tumor suppression protein. $5,8,10,11$ Mutation in the gene results in decreased gene expression and loss of function of E-cadherin. This leads to abnormal morphogenesis and architecture of epithelial tissue, loss of cellular polarity and contact inhibition, unregulated growth, and invasion of adjacent tissue. ${ }^{12,13}$

Approximately 50 distinct $\mathrm{CDH} 1$ mutations throughout the gene have been described..$^{2,11,13}$ In addition to mutations, DNA methylation of the CDH1 promoter has been observed and may completely deactivate the gene, decreasing expression of E-cadherin. ${ }^{13}$ Another recent observation is that when E-cadherin expression is greatly decreased or absent, exon v6-containing CD44 isoforms are over-expressed. ${ }^{14}$ Several CD44 isoforms are thought to play a role in malignant transformation. ${ }^{14}$ In normal gastric mucosa, exon v6-containing isoforms are rarely expressed, but in pre-malignant and malignant gastric mucosa their expression increases. ${ }^{14}$ Therefore, these isoforms could become biomarkers for detecting the development of cancer in gastric mucosa. ${ }^{14}$

Between $25 \%$ and $50 \%$ of families that fit the International Gastric Cancer Linkage Consortium (IGCLC) criteria for HDGC will have autosomal dominant inherited germline CDH1 mutations, , $^{2,6-11,13-15}$ and about $39 \%$ of HDGC patients carry a CDH1 gene mutation. ${ }^{7,8,13}$ The penetrance is approximately $75 \%, 4,7,8$ so the lifetime chance of an individual with a $\mathrm{CDH} 1$ mutation developing gastric cancer is great, and the risk increases with age. At age 20, the risk is estimated to be $<1 \%$, increasing to about $4 \%$ by age 30 . By age 50 , the risk is $21 \%$ for men and $46 \%$ for women, and by age 80 it is approximately $54 \%$ for men and $75 \%$ for women., ${ }^{2,-9,13,15,16}$ In addition to HDGC, individuals who carry a CDH1 mutation have an estimated $46 \%$ risk of developing lobular breast cancer $(\mathrm{LBC})$. $^{2,8,10,11}$

Diffuse gastric cancer is one of two main morphological types of gastric cancer, with intestinal-type being the other. Although both are gastric cancers, they differ in many ways. In a study by Janssen et al, ${ }^{17} 58 \%$ of their population had the intestinal type of gastric cancer, while $26 \%$ had the diffuse type, and $16 \%$ were unclassifiable. There was no difference in the rates of diffuse gastric cancer between men and women; however, the rate of intestinal-type gastric cancer in men was twice the rate in women. ${ }^{17}$ Within different age groups, the rates of each type of gastric cancer were consistent; however, in other studies it has been noted that diffuse gastric cancer is more predominant in younger patients, ${ }^{18}$ with the average age of diagnosis being 40 years, which is considered an early onset for most cancers. ${ }^{2,6-9,13,15,16}$ Genetically, relatives of patients with diffuse gastric cancer have a 7-fold increased risk of developing gastric cancer, compared to the 1.4-fold risk in relatives of patients with the intestinal type. ${ }^{18}$ The intestinal-type gastric cancer, most commonly found in the fundus, is generally well-differentiated due to preserved intracellular adhesion molecules causing the tumor cells to arrange in tubules or glands. ${ }^{18,19}$ It is highly associated with Helicobacter pylori infection, atrophy, and intestinal metaplasia. ${ }^{18,20}$ The apoptosis-related proteins, FAS and Bcl$\mathrm{xl}$, are expressed predominantly in the intestinal type of gastric cancer and can be used to differentiate it from the diffuse type. ${ }^{20}$

Diffuse gastric cancer is poorly-differentiated due to defective intracellular adhesion molecules caused by mutations in E-cadherin that are common in this type of gastric cancer. ${ }^{18,19}$ These defective intracellular adhesion molecules allow individual tumor cells to grow and invade neighboring structures without distinct formations. ${ }^{19,20}$ The presence of signet ring cells and mucin is occasionally seen in diffuse gastric cancer. ${ }^{20}$ Diffuse gastric cancer can be associated with H. pylori infection; however, it is not associated with atrophy or intestinal metaplasia. ${ }^{20}$

Early detection and diagnosis of HDGC are difficult because it is mostly located submucosally, and distributed as discrete foci along the entire length of the stomach. ${ }^{21}$ Currently, there are no reliable screening methods for early detection. Prognosis is generally poor, because this type of cancer is often found at a later stage, is more aggressive, and more difficult to treat. Advanced gastric cancer is not considered curable, so treatment is given for disease control and symptom palliation.

Due to the high risk of individuals with a CDH1 gene mutation developing HDGC, the difficulty in early detection, and the relatively early age of cancer onset, genetic testing availability can greatly impact a patient's family members. Recommendations regarding mutational analysis screening were made in 1999 by the IGCLC and are for those individuals with a minimum of two cases of diffuse gastric cancer in first and/or second degree relatives, with one diagnosis before age 50 , or three or more confirmed cases with onset at any age. ${ }^{10,22}$ These guidelines were updated to include individuals with diffuse gastric cancer diagnosed before age 45 and individuals or family members diagnosed with HDGC and LBC or signet ring colon carcinoma before age $50 .^{6,10}$ The genetic testing is typically accomplished by denaturing high-performance liquid chromatography and automated DNA sequencing. It should be followed by genetic counseling to discuss the results, any risks based on the results, and options for cancer surveillance. 
It may be distressing for family members who test positive for a $\mathrm{CDH} 1$ gene mutation, but they should be encouraged to share their results with family members, since genetic testing is available, and there are also IGCLC guidelines for the management of unaffected individuals. These include endoscopic examinations with screening biopsies every six months starting at age 20. ${ }^{22}$ Annual screening (mammogram and breast magnetic resonance imaging) for LBC beginning at age 35 is advised for female CDH1 mutation carriers. ${ }^{10,16}$ Our case had a family history of only one known diagnosed gastric cancer; whereas other reported cases had more extensive family histories with multiple family members diagnosed with gastric cancer. ${ }^{8,11,23,24}$ This shows the importance of informing family members about positive mutation results, since a thorough, informative family history can potentially lead to faster testing, diagnosis, and treatment.

Endoscopy as a surveillance option, however, is unreliable because often patients with normal endoscopy and negative biopsies who choose prophylactic gastrectomy will have multiple sites of diffuse foci of tumor during review of postsurgical specimens. ${ }^{4,6,16}$ This may be due to the fact that an endoscopic biopsy may be taken too superficially and/or from a site where no cancerous foci are present or where many mucin-containing cells are located, causing the biopsy to be assessed as negative for cancer. Our case illustrates this limitation; family members had negative biopsies from screening endoscopies, but post-surgical gastrectomy specimens were positive for cancer.

Other approaches to early detection have been attempted. Chromoendoscopy and PET/CT have shown some potential for identifying early stage diffuse gastric cancer, however, both have limitations that prevent them from becoming an alternative to prophylactic gastrectomy. ${ }^{7}$ Chromoendoscopy, the use of a particular stain combination to selectively visualize carcinomas during endoscopy, ${ }^{2}$ had slightly better study results than routine endoscopy ${ }^{2}$ however, these have not been reproducible at other institutions, ${ }^{25}$ and both stains have been withdrawn from clinical use due to possible embryotoxicity and carcinogenic potential. ${ }^{2}$ PET scans can frequently be negative due to the mucin present in the neoplastic cells, ${ }^{26}$ and endoscopic ultrasound has not been helpful in early detection. ${ }^{6}$

Prophylactic gastrectomy is another form of management for individuals carrying a CDH1 gene mutation. This surgery may be a life-saving option because it eliminates the risk of developing diffuse gastric cancer and the need to undergo cancer surveillance methods that remain difficult and unreliable. ${ }^{7,8,15,27}$ However, despite the fact that the average age of onset of HDGC is about 40 years, total gastrectomy is usually only advised for those patients over age 20. Although gastrectomy provides a definitive treatment, it can involve significant morbidity and mortality. The mortality rate ranges from $0 \%$ to $7 \%$, and almost all gastrectomy patients develop a significant morbidity., ${ }^{2,716}$ However, a recent study examining the utility and safety of laparoscopic total gastrectomy showed that this technique decreased mortality and morbidity rates in their population, ${ }^{7}$ thereby offering a potentially safer surgical option in the near future.

Post-gastrectomy morbidities include malabsorption, diarrhea, malnutrition, and dumping syndrome, leading to weight loss and multiple vitamin and mineral deficiencies. ${ }^{2,7}$ Although most morbidities are initially severe, they tend to improve over time, especially with appropriate diet modification and nutritional counseling. ${ }^{2}$ Those HDGC patients who choose prophylactic gastrectomy should be managed with a multidisciplinary approach, providing preoperative team counseling including a gastroenterologist, surgeon, dietician, genetic counselor, and specialist nurse ${ }^{6,8}$ Patients should be well informed about potential surgical complications, post-gastrectomy morbidities, and changes in diet that will likely occur, so they can make the most appropriate management decision.

In conclusion, HDGC is a rare inherited cancer, with onethird to one-half of cases due to a mutation in the $\mathrm{CDH} 1$ gene. Carriers of the mutation have an approximate $75 \%$ lifetime risk of developing HDGC. Surveillance by upper endoscopy is ineffective and unreliable; therefore, total prophylactic gastrectomy is advised after age 20 . Though this procedure is life-saving, it has significant morbidities that require management by a multidisciplinary team. As our case highlights, an early, in-depth evaluation of family history upon presentation of certain symptoms could lead to earlier genetic testing and diagnosis, and an HDGC diagnosis should lead to prompt genetic evaluation of other family members followed by preventive measures, including surgeries in affected members, due to the high penetrance of the gene.

\section{Acknowledgements}

The authors thank Thanhcuong T. Nguyen, MD of the Marshfield Clinic Pathology Department for providing the pathology photos and explanations, and the Marshfield Clinic Research Foundation's Office of Scientific Writing and Publication for assistance in the preparation of this case report.

\section{References}

1. Kamangar F, Dores GM, Anderson WF. Patterns of cancer incidence, mortality and prevalence across five continents: defining priorities to reduce cancer disparities in different geographic regions of the world. J Clin Oncol 2006; 24:2137-2150.

2. Pandalai PK, Lauwers GY, Chung DC, Patel D, Yoon SS. Prophylactic total gastrectomy for individuals with germline CDH1 mutation. Surgery 2011;149:347-355.

3. National Cancer Institute. A snapshot of stomach cancer. 2011. Available at: http://www.cancer.gov/aboutnci/servingpeople/ snapshots/stomach.pdf. Accessed April 6, 2012.

4. Lynch HT, Kaurah P, Wirtzfeld D, Rubinstein WS, Weissman S, Lynch JF, Grady W, Wiyrick S, Senz J, Huntsman DG. Hereditary diffuse gastric cancer: diagnosis, genetic counseling and prophylactic total gastrectomy. Cancer 2008;112:2655-2663. 
5. Ghaffari SR, Rafati M, Sabokbar T, Dastan J. A novel truncating mutation with the E-cadherin gene in the first Iranian family with hereditary diffuse gastric cancer. Eur J Surg Oncol 2010;36:559-562.

6. Fitzgerald RC, Hardwick R, Huntsman D, Carneiro F, Guilford P, Blair V, Chung DC, Norton J, Ragunath K, Van Krieken JH, Dwerryhouse S, Caldas C; International Gastric Cancer Linkage Consortium. Hereditary diffuse gastric cancer: updated consensus guidelines for clinical management and directions for future research. J Med Genet 2010;47:436-444.

7. Hackenson D, Edelman DA, McGuire T, Weaver DW, Webber JD Prophylactic laparoscopic gastrectomy for hereditary diffuse gastric cancer: a case series in a single family. JSLS 2010;14:348-352.

8. Wilcox R, Perpich M, Noffsinger A, Posner MC, Cooper K Hereditary diffuse gastric cancer: multidisciplinary case report with review of the literature. Patholog Res Int 2011;2011:845821.

9. Oliveira C, Seruca R, Carneiro F. Genetics, pathology and clinics of familial gastric cancer. Int J Surg Pathol 2006;14:21-33.

10. Jakubowska A, Lawniczak M, Wojnarska B, Cybulski C, Huzarski T, Byrski T, Tołoczko-Grabarek A, Jaworska K, Durda K, Starzyńska T, Lubiński J. CDH1 gene mutations do not contribute in hereditary diffuse gastric cancer in Poland. Fam Cancer 2010;9:605-608.

11. Matsukuma KE, Mullins FM, Dietz L, Zehnder JL, Ford JM, Chun NM, Schrijver I. Hereditary diffuse gastric cancer due to a previously undescribed $\mathrm{CDH} 1$ splice site mutation. Hum Pathol 2010;41:1200-1203.

12. Graziano F, Humar B, Guilford P. The role of the E-cadherin gene $(\mathrm{CDH} 1)$ in diffuse gastric cancer susceptibility: from the laboratory to clinical practice. Ann Oncol 2003; 14:1705-1713.

13. Yamamoto E, Hiromu S, Takamaru H, Yamamoto H, Toyota M, Shinomura Y. Role of DNA methylation in the development of diffuse-type gastric cancer. Digestion 2011;83:241-249.

14. da Cunha CB, Oliveira C, Wen X, Gomes B, Sousa S, Suriano G, Grellier M, Huntsman DG, Carneiro F, Granja PL, Seruca R. De novo expression on CD44 variants in sporadic and hereditary gastric cancer. Lab Invest 2010;90:1604-1614.

15. Suriano G, Yew S, Ferreira P, Senz J, Kaurah P, Ford JM, Longacre TA, Norton JA, Chun N, Young S, Oliveira MJ, Macgillivray B, Rao A, Sears D, Jackson CE, Boyd J, Yee C, Deters C, Pai GS, Hammond LS, McGivern BJ, Medgyesy D, Sartz D, Arun B, Oelschlager BK, Upton MP, NeufeldKaiser W, Silva OE, Donenberg TR, Kooby DA, Sharma S, Jonsson BA, Gronberg H, Gallinger S, Seruca R, Lynch H, Huntsman DG. Characterization of a recurrent germline mutation of the E-cadherin gene: implications for genetic testing and clinical management. Clin Cancer Res 2005;11:5401-5409.

16. Blair V, Martin I, Shaw D, Winship I, Kerr D, Arnold J, Harawira P, McLeod M, Parry S, Charlton A, Findlay M, Cox B, Humar B, More H, Guilford P. Hereditary diffuse gastric cancer: diagnosis and management. Clin Gastroenterol Hepatol 2006;4:262-275.

17. Janssen CW, Maartmann-More H, Lie RT, Matre R. Age and sex distribution of intestinal type and diffuse gastric cancer. Acta Patholigica, Microbiologica et Immunologica Scandinavica 1991;99:78-82.

18. Bevan S, Houlston RS. Genetic predisposition of gastric cancer. Q J Med 1999;92:5-10.

19. Correa P. Pathology and molecular pathogenesis of gastric cancer. Up to Date.com Web site, January 2011. Available at http://www.uptodate.com/contents/pathology-and-molecularpathogenesis-of-gastric-cancer?source=search_result\&search $=$ Pathology + and + molecular + pathogenesis + of + gastric + cancer .+2011\&selectedTitle=1\%7E150. Accessed February 7, 2012.
20. van der Woude CJ, Kleibeuker JH, Tiebosch ATGM, Homan M, Beuving A, Jansen PLM, Moshage H. Diffuse and intestinal type gastric carcinomas differ in their expression of apoptosis related proteins. J Clin Pathol 2003;56:699-702.

21. Moran CJ, Joyce M, McAnena OJ. CDH1 associated gastric cancer: a report of a family and review of the literature. Eur J Surg Oncol 2005;31:259-264.

22. Caldas C, Carneiro F, Lynch HT, Yokota J, Wiesner GL, Powell SM, Lewis FR, Huntsman DG, Pharoah PD, Jankowski JA, MacLeod P, Vogelsang H, Keller G, Park KG, Richards FM, Maher ER, Gayther SA, Oliveira C, Grehan N, Wight D, Seruca R, Roviello F, Ponder BA, Jackson CE. Familial gastric cancer: overview and guidelines for management. J Med Genet 1999;36:873-880.

23. Francis WP, Rodrigues DM, Perez NE, Lonardo F, Weaver D, Webber JD. Prophylactic laparoscopic-assisted total gastrectomy for hereditary diffuse gastric cancer. J Society Laparoendoscopic Surgeons 2007;11:142-147.

24. Van Domselaar F, Correa D, Vaccaro C, Redal M, Van Domselaar R, Huntsman D, Kaurah P, Senz J, Lynch H. [Hereditary diffuse gastric cancer (HDGC): presentation of a family with a new mutation of the CDH1 gene]. [Article in Spanish] Acta Gastroenterol Latinoam 2007;37:158-163.

25. Shaw D, Blair V, Framp A, Harawira P, McLeod M, Guilford P, Parry S, Charlton A, Martin I. Chromoendoscopic surveillance in hereditary diffuse gastric cancer: an alternative to prophylactic gastrectomy? Gut 2005; 54:461-468.

26. Berger KL, Nicholson SA, Dehdashti F, Siegel BA. FDG PET evaluation of mucinous neoplasms: Correlation of FDG uptake with histopathologic features. AJR Am J Roentgenol. 2000;174:1005-1008.

27. Bacani JT, Soares M, Zwingerman R, di Nicola N, Senz J, Riddell R, Huntsman DG, Gallinger S. CDH1/E-cadherin germline mutations in early-onset gastric cancer. J Med Genet 2006;43:867-872.

\section{Author Affiliations}

Adedayo A. Onitilo, MD, MSCR, FACP*;

Govinda Aryal, MD $;$; Jessica M. Engel, MS, RN"

*Department of Hematology/Oncology, Marshfield Clinic

Weston Center, Weston, WI, USA

tDepartment of Internal/Hospital Medicine, Marshfield

Clinic, Marshfield, WI, USA

\#Department of Oncology, Marshfield Clinic at Ministry

St. Michael's Hospital, Stevens Point, WI, USA 